Progress of Theoretical Physics, Vol. 120, No. 5, November 2008

\title{
Phenomenology of high gluon density QCD and heavy-ion physics at ISMD 2011: $x$ smaller than ever !
}

\author{
Adrian Dumitru ${ }^{1,2 *)}$ \\ ${ }^{1}$ RIKEN BNL Research Center, Brookhaven National Laboratory \\ Upton, NY 11973, USA \\ ${ }^{2}$ Department of Natural Sciences, Baruch College, CUNY \\ 17 Lexington Avenue, New York, NY 10010, USA
}

(Received April 1, 2004; revised November 20, 2008)

\begin{abstract}
I provide a brief summary of the theory presentations at ISMD 2011 related to the phenomenology of small- $x$ QCD evolution and its application to particle production and fluctuations in high-energy hadron and heavy-ion collisions. I also mention some challenges for quantitative phenomenology which emerged from the LHC, such as understanding the long-range "ridge" in high-multiplicity $p+p$ collisions, the transverse momentum distributions in $p+p$ at semi-hard $p_{\perp}$, and the origin and scale of density fluctuations in the initial state of heavy-ion collisions.
\end{abstract}

\section{$\S 1$. Introduction}

The physics of multi-particle dynamics has a long history. Indeed, ISMD 2011 on Miyajima-Island, Hiroshima, was the $41^{\text {st }}$ meeting in the International Symposium on Multiparticle Dynamics series. The talks have mostly focused on physics at accelerator facilities, in particular at the BNL-RHIC and CERN-LHC colliders. They covered a very broad range of topics including particle production and multiparticle correlations in high-energy collisions, studies of hard QCD jets and of the underlying event, collective effects in QCD at high temperatures and baryon densities, femtoscopic Bose-Einstein correlations, as well as electroweak physics of the standard model and beyond. However, we had talks also on cosmic ray physics and its relation to accelerator based high-energy QCD, and finally on strong-field QED with high intensity lasers. It is, indeed, a time of exciting progress in this field and ISMD 2011 provided a nice overview as well as a platform for stimulating discussions.

This write-up is intended to offer a brief summary of the theory presentations at ISMD 2011 related to small-x QCD; more specifically, of the phenomenology of small- $x$ QCD evolution and its application to particle production and fluctuations in high-energy hadron and heavy-ion collisions.

\section{$\S 2$. Phenomenology of running-coupling BK evolution}

Collisions of protons or heavy ions such as lead at high energies release a very large number of gluons from their wave functions which dominate the production of new particles and anti-particles. In fact, the wave function of a hadron boosted to

*) e-mail: Adrian.Dumitru@baruch.cuny.edu 
(nearly) the light cone is so densely packed with gluons that they may "overlap", leading to non-linear interactions. ${ }^{1)}$ Therefore, at high energies the colliding hadrons can be treated as a high occupancy gluon field. This dense system is nowadays referred to as a Color Glass Condensate (CGC). For a recent in-depth review of the CGC we refer to ref. ${ }^{2)}$
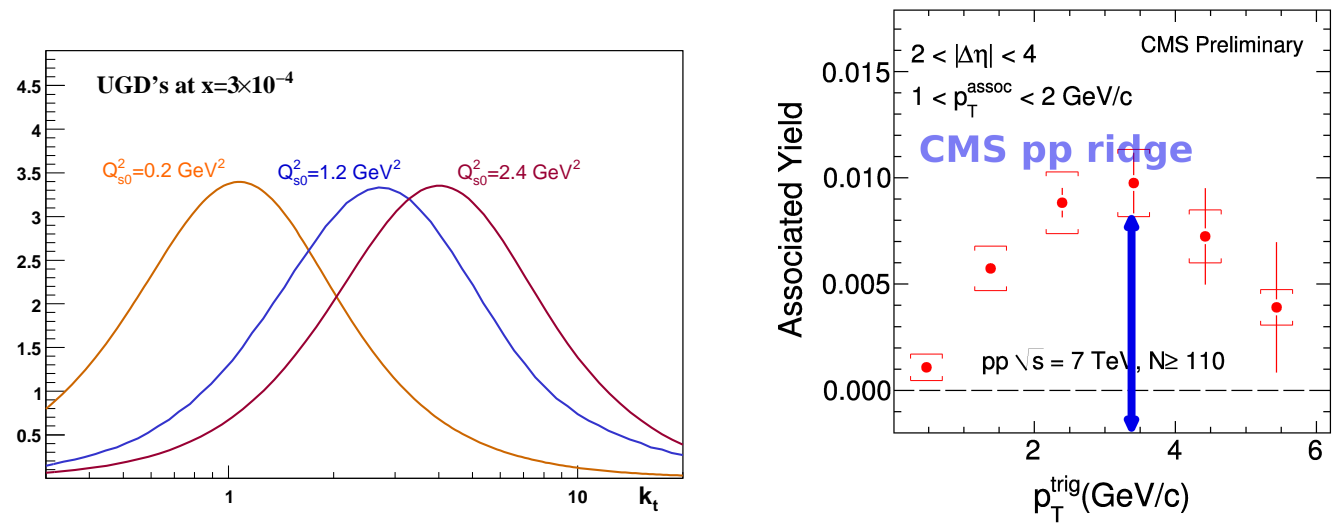

Fig. 1. Left: Albacete's unintegrated gluon distribution $\Phi\left(k_{\perp}, x\right)$ obtained from rcBK evolution of the MV-model dipole, eq. $(2 \cdot 4)$. Right: associated yield in CMS ridge peaks about a semi-hard scale.

The evolution of observables with energy is described by "small- $x$ " QCD evolution equations (where $x \sim 1 / \sqrt{ } s$ is inversely proportional to the energy of the beams). The two-point function of two light-like Wilson lines, in particular,

$$
\mathcal{N}(r, x)=1-\frac{1}{N_{c}}\left\langle\operatorname{tr} V_{r} V_{0}^{\dagger}\right\rangle
$$

determines DIS structure functions and single-inclusive cross sections in hadronic collisions $^{*}$. $\mathcal{N}(r, x)$ represents the scattering amplitude of a color dipole of transverse size $r$. Its evolution with energy is governed by the non-linear so-called BalitskyKovchegov (BK) equation,

$\frac{\partial \mathcal{N}(r, x)}{\partial \log \left(x_{0} / x\right)}=\int d^{2} r_{1} K\left(r, r_{1}, r_{2}\right)\left[\mathcal{N}\left(r_{1}, x\right)+\mathcal{N}\left(r_{2}, x\right)-\mathcal{N}(r, x)-\mathcal{N}\left(r_{1}, x\right) \mathcal{N}\left(r_{2}, x\right)\right]$

Present numerical solutions (and corresponding applications) of this evolution equation employ a kernel valid to running coupling accuracy, $\left.{ }^{4}\right)$

$$
K\left(r, r_{1}, r_{2}\right)=\frac{N_{c} \alpha_{s}\left(r^{2}\right)}{2 \pi^{2}}\left[\frac{1}{r_{1}^{2}}\left(\frac{\alpha_{s}\left(r_{1}^{2}\right)}{\alpha_{s}\left(r_{2}^{2}\right)}-1\right)+\frac{r^{2}}{r_{1}^{2} r_{2}^{2}}+\frac{1}{r_{2}^{2}}\left(\frac{\alpha_{s}\left(r_{2}^{2}\right)}{\alpha_{s}\left(r_{1}^{2}\right)}-1\right)\right] .
$$

From the Fourier transform of the scattering amplitude for a dipole in the adjoint representation, $\mathcal{N}_{A}(r, x)=2 \mathcal{N}(r, x)-\mathcal{N}^{2}(r, x)$, one obtains the "(dipole) unintegrated

*) On the other hand, the connected contribution to multi-particle production in general involves higher $n$-point functions. 
gluon distribution" $\Phi\left(k_{\perp}, x\right) \sim k_{\perp}^{2} \mathcal{N}\left(k_{\perp}, x\right)$ which determines the single-inclusive gluon production cross section. Owing to the non-linear term in $(2 \cdot 2)$ this function vanishes as $k_{\perp} \rightarrow 0$ and peaks about a semi-hard scale $\sim Q_{s}(x)$, as shown in fig. 1 . Although the direct analogy (so far) is somewhat murky, it is nevertheless tempting to show it side by side with the "ridge yield" obtained by CMS for high-multiplicity $\mathrm{p}+\mathrm{p}$ collisions at $7 \mathrm{TeV}^{5}$ ) (also shown by J.-H. Kim at this meeting); the associated yield in the ridge evidently peaks about a semi-hard scale, too, which needs to be understood more clearly (for some initial ideas to relate the CMS ridge to the properties of non-linear evolution see refs. ${ }^{6)}$ ).

As shown in the talks by Albacete, Fujii and Nara, the rcBK solution also provides a good description of HERA DIS structure functions at low $x$, of hadron transverse momentum distributions in the forward region of $p+p$ and $d+A u$ collisions at RHIC, and of $p_{\perp}$-integrated hadron multiplicities in $P b+P b$ collisions at the LHC.
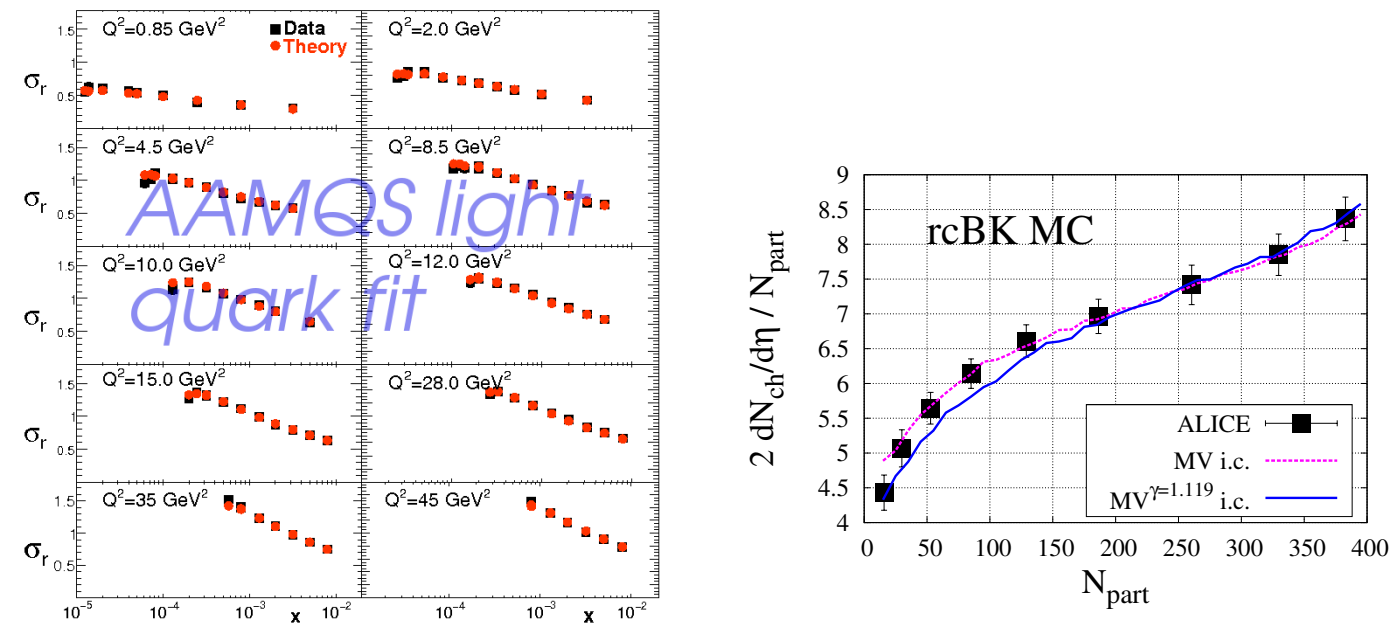

Fig. 2. Left: AAMQS rcBK fit to HERA structure functions. ${ }^{7)}$ Right: charged hadron multiplicities versus centrality for $\mathrm{Pb}+\mathrm{Pb}$ collisions at $2.76 \mathrm{TeV}{ }^{8)}$ Presented by J. Albacete.

Some examples are shown in figs. 2 and 3. It is worth noting though that the AAMQS DIS fit requires an initial condition for eq. $(2 \cdot 2)$ which is not like the "MV model" dipole,

$$
\mathcal{N}_{M V}\left(r, x_{0}\right)=1-\exp \left(-\frac{1}{4} r^{2} Q_{s}^{2}\left(x_{0}\right) \log \frac{1}{r \Lambda}\right),
$$

but one which falls off more rapidly at small $r$ :

$$
\mathcal{N}_{M V^{\gamma}}\left(r, x_{0}\right)=1-\exp \left(-\frac{1}{4}\left(r^{2} Q_{s}^{2}\left(x_{0}\right)\right)^{\gamma} \log \frac{1}{r \Lambda}\right) \quad, \quad(\gamma>1) .
$$

This finding is underlined by the charged hadron transverse momentum distributions in $p+p$ collisions measured at the LHC, see fig. 4 . The transverse momentum distributions at $p_{\perp}>Q_{s}$ have been obtained by first computing the transverse momentum distribution of gluons from the $k_{\perp}$-factorization formula which is then 

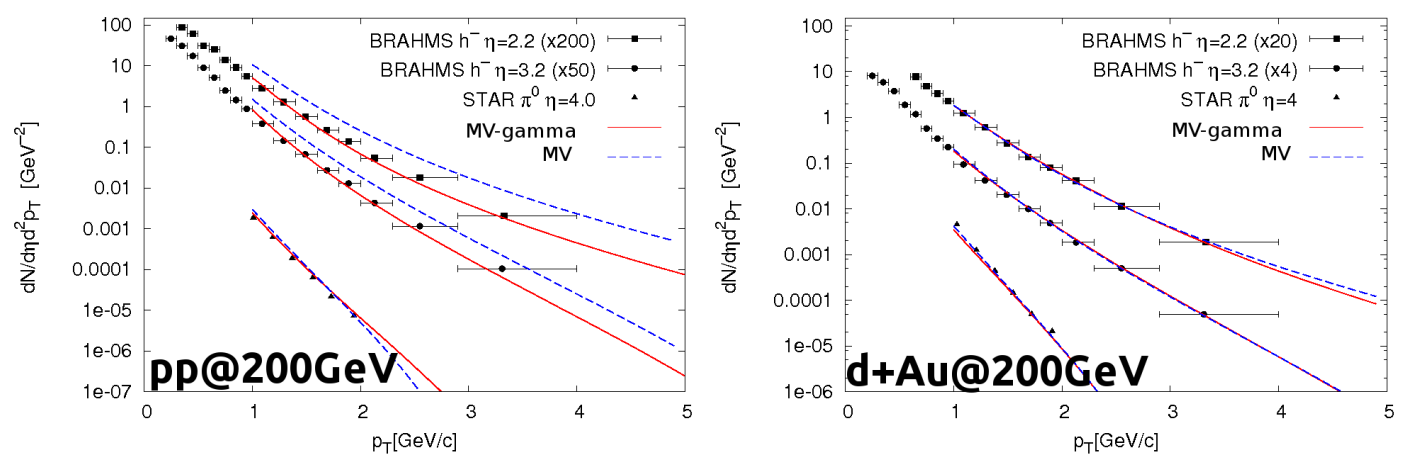

Fig. 3. Hadron transverse momentum distributions in the forward region of $p+p$ and $d+A u$ collisions at RHIC as presented by H. Fujii at this meeting. ${ }^{9)}$

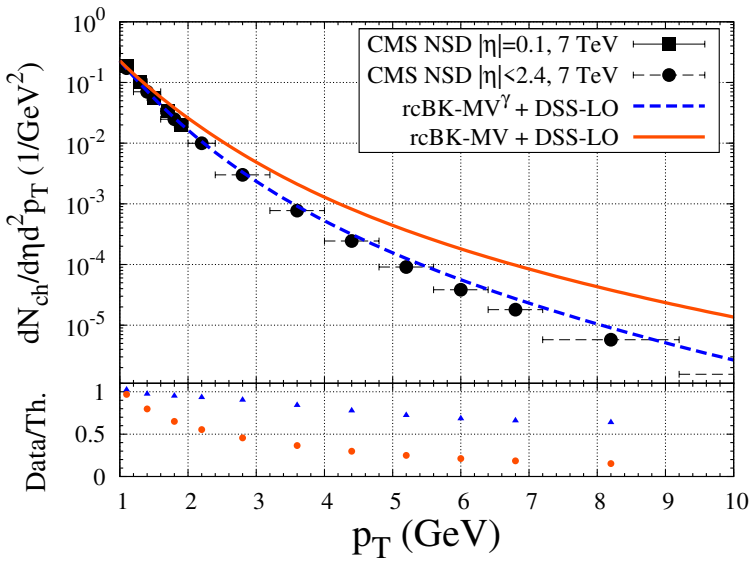

Fig. 4. Hadron transverse momentum distributions in the central region of $p+p$ collisions at $7 \mathrm{TeV} .{ }^{10)}$ Theory $K$ factor adjusted to fit data at $p_{\perp}=1 \mathrm{GeV}$. CMS data from. ${ }^{11)}$

convoluted with a DSS gluon $\rightarrow$ hadron QCD fragmentation function: ${ }^{12}$

$$
\frac{d N_{h^{ \pm}}}{d y d^{2} p_{\perp}}=\int \frac{d z}{z^{2}} D_{g}^{h^{ \pm}}\left(z, Q^{2}\right) \frac{d N_{g}}{d y d^{2} q_{\perp}} .
$$

Here, $p_{\perp}=z q_{\perp}$ is the transverse momentum of the produced hadron and the scale $Q^{2}$ may be chosen as $q_{\perp}^{2}$. For the first time, the extreme energy reach of LHC enables us to perform quantitative tests of the theory at high transverse momentum $p_{\perp}$ but small $x$. Evidently, the MV model initial condition with rcBK evolution (plus $k_{\perp}$ factorization) disagrees with the CMS data. On the other hand, it is comforting to see that the AAMQS-modified $\mathrm{MV}^{\gamma}$ initial condition which worked for $F_{2}$ at HERA provides a good match to the LHC high- $p_{\perp}$, low- $x$ data as well.

As is often the case, answers lead to more questions. Such as: is there any theory which would at least motivate the successful AAMQS guess (2.5) ? The MV model initial condition $(2 \cdot 4)$ can be derived from an effective theory treating the large- $x$ valence charges as recoilless sources on the light cone (analogous to the Weizsäcker- 
Williams equivalent photon approximation); in the limit of a very large number of such valence charges, color fluctuations are described by a quadratic action ${ }^{13}$ )

$$
S=\int d^{2} x_{\perp} \frac{1}{2 \mu^{2}} \rho^{a} \rho^{a}
$$

where $\mu^{2}\left(x_{\perp}\right) \sim N_{v a l}$ is proportional to the number of valence charges at a given transverse coordinate. For a nucleus, $N_{v a l} \sim N_{c} A^{1 / 3}$ is proportional to the number of colors and to the longitudinal thickness.

The $\mathrm{MV}^{\gamma}$ initial condition $(2 \cdot 5)$, on the other hand, has not been derived (so far) from an effective action for the large- $x$ valence charges. Worse yet, there is no theoretical understanding of the dependence of the parameter $\gamma$ on $N_{v a l}$ resp. on the thickness $A^{1 / 3}$ of a nucleus; $\gamma$ would have to be re-fitted for every single hadron or nucleus. Understanding the $A$ dependence of the AAMQS parameter $\gamma$ will also be important for

i) the ratio of $p A$ to $p p$ spectra at the LHC: some estimates using rcBK unintegrated gluon densities suggest that $R_{p A}$ might be suppressed at LHC energies, even away from the proton fragmentation region, due to the effects from small- $x$ evolution; ${ }^{15}$ ) and

ii) for predictions or fits of structure functions of heavy ions at small $x$ which will hopefully be measured by a future electron-ion collider eIC. ${ }^{14)}$

One possibility is that the AAMQS initial condition arises from corrections to the MV model action which involve higher powers of the color charge density $\rho$. Such terms are allowed by $\mathrm{SU}(\mathrm{N})$ invariance and involve couplings suppressed by additional powers of $1 / g A^{1 / 3}$ :

$$
S=\int d^{2} x_{\perp}\left[\frac{1}{2 \mu^{2}} \rho^{a} \rho^{a}-\frac{1}{\kappa_{3}} d^{a b c} \rho^{a} \rho^{b} \rho^{c}+\frac{1}{\kappa_{4}} \rho^{a} \rho^{a} \rho^{b} \rho^{b}\right] .
$$

Here, $\mu^{2} \sim g^{2} A^{1 / 3}, \kappa_{3} \sim g^{3} A^{2 / 3}$, and $\kappa_{3} \sim g^{4} A$. ${ }^{16)}$ This action would allow for definite predictions for the $A$-dependence of the initial condition for rcBK evolution. It will be interesting to see if $(2 \cdot 8)$ is able to reproduce the essential features of the AAMQS MV ${ }^{\gamma}$ model.

Coming back to

$$
R_{p A}\left(p_{\perp}\right)=\frac{d N_{p A} / d^{2} p_{\perp}}{N_{\text {coll }} d N_{p p} / d^{2} p_{\perp}},
$$

I. Potashnikova and B. Kopeliovich in their contribution pointed out that there are some subtleties in defining the "number of binary parton-parton collisions" $N_{\text {coll. }}{ }^{17)}$ This is commonly taken as

$$
N_{\text {coll }}=\frac{\sigma_{\text {in }}^{p p}\left\langle T_{A}\right\rangle}{\left\langle P_{\text {in }}\right\rangle}
$$

where $T_{A}$ denotes the thickness function of the target nucleus, $P_{\text {in }}$ is the probability for an inelastic $p A$ interaction, and $\langle\cdot\rangle$ denotes an average over events. The authors argued that the cross section in eq. $(2 \cdot 10)$ should be corrected for the fraction of missed diffractive events. If this represents a significant fraction then $N_{\text {coll }}$ is reduced and this would affect our interpretation of nuclear effects as measured by $R_{p A}$. 

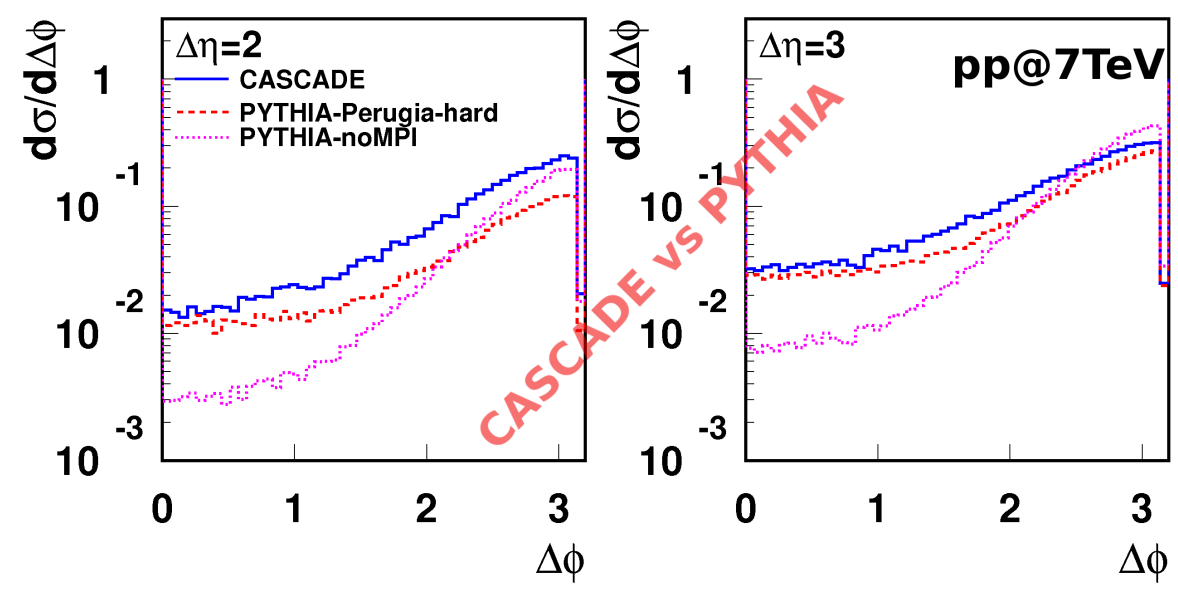

Fig. 5. Cross section for two jets with $p_{\perp}>10 \mathrm{GeV}$ separated by a rapidity interval $\Delta \eta$ and azimuthal angle $\Delta \phi ; p+p$ collisions at $\sqrt{ } s=7 \mathrm{TeV}$. From ref. ${ }^{18)}$

Another important test for our present knowledge of the unintegrated gluon densities has been emphasized by K. Kutak at this meeting. That is, the singleinclusive cross section at non-central rapidities where the evolution is asymmetric; also, the angular distribution of semi-hard jets separated by a rapidity interval $\Delta \eta^{18}$ ) shown in fig. 5 .

\section{§3. Density fluctuations in $p+p$ and $A+A$ collisions}

There has been a lot of interest recently in understanding the magnitude and length scale of fluctuations of the density of produced gluons in the transverse plane; several presentations at this meeting addressed related questions. In their talks, A. Mocsy and P. Sorensen noted the analogy to the power spectrum of CMB fluctuations which provides information on the evolution of the early universe. An artists view is shown in fig. 6 . They presented the harmonic spectrum of the initial density distribution (preceding the hydrodynamic expansion in $A+A$ collisions) in terms of the eccentricities

$$
\epsilon_{n}^{2}=\frac{\left\langle r^{2} \cos n \phi\right\rangle^{2}+\left\langle r^{2} \sin n \phi\right\rangle^{2}}{\left\langle r^{2}\right\rangle^{2}}
$$

$\langle\cdot\rangle$ denotes an average over the initial distribution of produced gluons in the transverse plane, ${ }^{20)} d N / d \eta d^{2} r$. One commonly considered source of inhomogeneities is due to the fluctuations of the positions of the nucleons in the nuclei before the collision. In the absence of a length scale (such as a non-zero radius of the nucleon) for these fluctuations, and for central collisions, Sorensen argues that the spectrum of $\epsilon_{n}$ is flat*).

\footnotetext{
*) The peak of $\epsilon_{2}^{2}$ in fig. 6 for $b=0$ collisions is due to the intrinsic deformation of a $A u$ nucleus.
} 


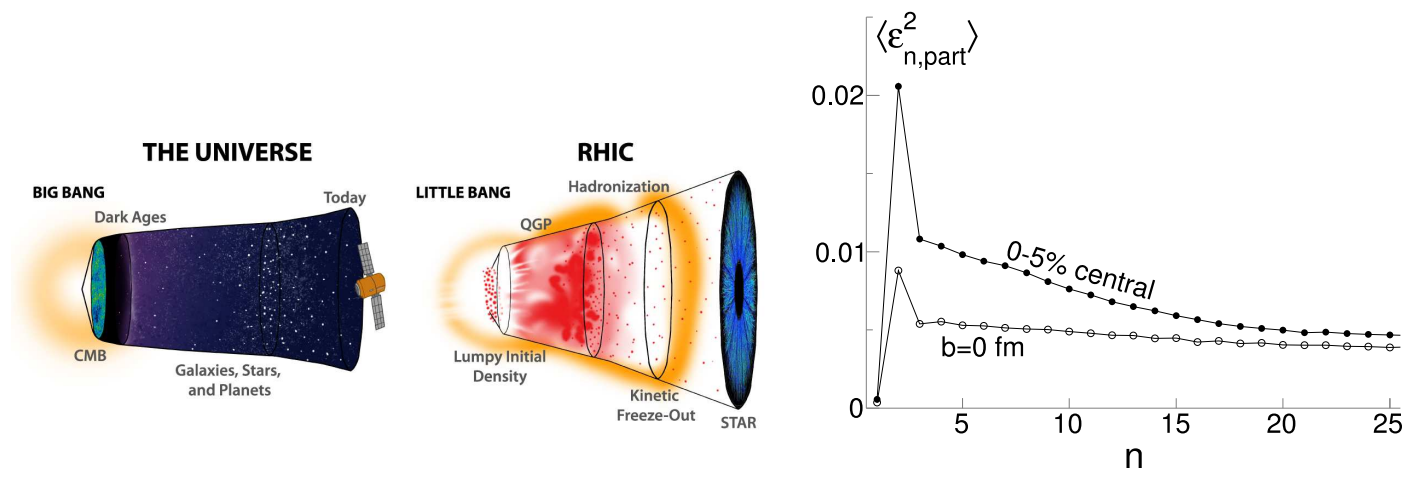

Fig. 6. Left: Artists view of the expansion of the universe and of the survivial of initial quantum fluctuations as CMB fluctuations measured today; and of the expansion of fluctuations in $p+p$ and $A+A$ collisions. ${ }^{19)}$ Figure by A. Doig. Right: harmonic spectrum of density inhomogeneities in central $A+A$ collisions for point-like nucleons. Presented at ISMD 2011 by A. Mocsy and P. Sorensen.

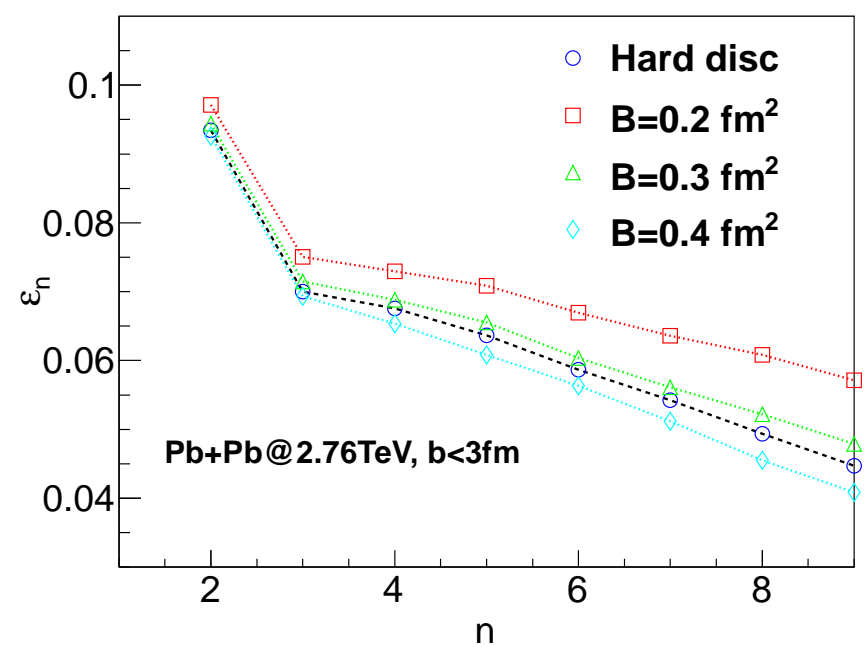

Fig. 7. Right: Eccentricity spectrum averaged over Monte-Carlo ensembles of nucleon configurations with Gaussian valance parton distributions, obtained from $k_{\perp}$ factorization with $\mathrm{rcBK}$ unintegrated gluon densities. Presented by Y. Nara. ${ }^{21)}$

Y. Nara showed the Fourier spectrum of eccentricities obtained from a simulation of heavy-ion collisions based on the $k_{\perp}$ factorization formula with rcBK unintegrated gluon densities, averaged over an ensemble of configurations of the colliding nuclei. Again, here the only source of fluctuations are the random positions of the nucleons before the collision (the average is a Woods-Saxon distribution); they determine the locations of the valence charges for small- $x$ evolution. The valence charge profile function was assumed to be Gaussian rather than a delta-function. The Fourier spectrum of eccentricities then drops towards higher harmonics, the faster the bigger the Gaussian width $B$. A careful understanding of higher harmonics, in particular of those moments which are dominated by fluctuations, will require constraints on 
the Gaussian width $B$ from exclusive vector meson production in DIS, and from multiplicity distributions in $p+p$ and $p+A$; see, e.g., ref. ${ }^{22)}$ and references therein.

Fluctuations of the nucleon configurations in the colliding nuclei are very likely not the full story, however, as has been pointed out by C. Flensburg and Y. Hatta. There are intrinsic fluctuations of the number of emitted gluons even for a fixed number of longitudinally stacked projectile and target nucleons (or "participants") which are actually amplified in a collision of strong chromo fields. ${ }^{23)}$ Furthermore, one expects fluctuations due to stochastic dipole splitting events as implemented in Flensburg's DIPSY Monte-Carlo; ${ }^{24)}$ the probability distribution for splitting is given by

$$
d P=\alpha_{s} \frac{N_{c}}{2 \pi^{2}} \frac{(\vec{x}-\vec{y})^{2}}{(\vec{x}-\vec{z})^{2}(\vec{z}-\vec{y})^{2}} d^{2} z d Y,
$$

if a fixed coupling constant $\alpha_{s}$ is assumed. Hatta stressed that $(3 \cdot 2)$ results in large fluctuations of the number of dipoles which are strongly correlated in the impact parameter plane.

In contrast, so far solutions of the rcBK equation have been averaged over all random emissions, and corresponding particle production models have not considered the effects from this type of fluctuations. Fig. 8 shows that fluctuations in dipole splittings can increase $\epsilon_{3}$ quite significantly, while $\epsilon_{2}$ appears to be largely unaffected.
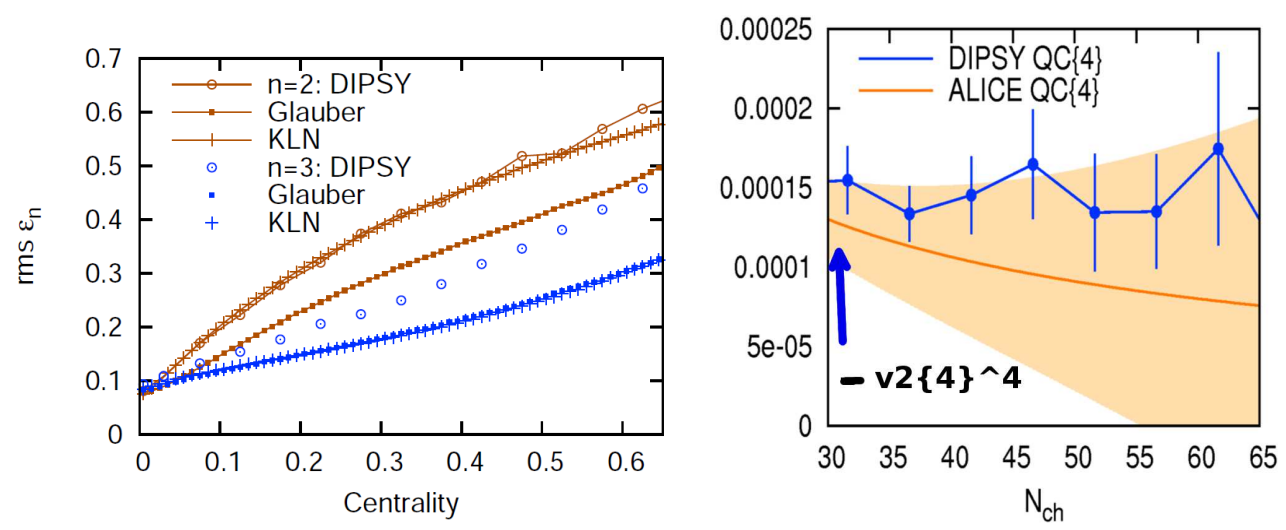

Fig. 8. Left: $\epsilon_{n}$ from the Monte-Carlo stochastic dipole splitting model DIPSY, from a geometric "wounded nucleon / Glauber" model, and from Nara's MC-KLN model ${ }^{26)}$ (fluctuations of large$x$ sources only). Presented by C. Flensburg. Right: DIPSY prediction for the fourth cumulant of non-flow correlations for high-multiplicity $p+p$ collisions at $7 \mathrm{TeV}$. Preliminary ALICE data from. ${ }^{27)}$ Presented by Y. Hatta.

The presence of such large fluctuations in the gluon density is evident from the multiplicity distribution in $p+p$ collisions at high energies. It also makes it feasible to define Fourier "eccentricities" of the transverse area occupied by produced gluons in high-multiplicity $p+p$ collisions, in analogy to heavy-ion collisions. What is measured though are the momentum-space azimuthal anisotropies $v_{n}$ in the final state. DIPSY predicts that the large fluctuations would lead to $v_{2}^{4}\{4\}$ (measured via four-particle cumulants) which is negative, corresponding to "non-flow" correlations. ${ }^{25)}$ 


\section{$\S 4$. Summary}

With the ongoing program (and possible upgrades) at RHIC and with the recent advent of LHC, this is clearly an excellent time for progress in high-energy QCD. Tools have been constructed, and constantly refined, to address questions about the strong color fields of hadrons and nuclei boosted nearly to the light cone and their manifestation in particle production and in fluctuations analogous to those at the Big Bang. Hopefully measurements at non-central rapidities as well as for $p+A$ collisions will be possible at the LHC to probe QCD in a highly non-linear regime.

\section{Acknowledgements}

It is a great pleasure to thank the organizers of ISMD 2011 for the opportunity to visit Miyajima-island (Hiroshima) and to participate in this interesting multi-faceted conference! Also, I gratefully acknowledge support by the DOE Office of Nuclear Physics through Grant No. DE-FG02-09ER41620 and for PSC-CUNY Award 633820042, jointly funded by The Professional Staff Congress and The City University of New York.

\section{References}

1) L. V. Gribov, E. M. Levin and M. G. Ryskin, Phys. Rep. 100 (1983), 1. A. H. Mueller and J.-W. Qiu, Nucl. Phys. B 268 (1986), 427.

2) F. Gelis, E. Iancu, J. Jalilian-Marian and R. Venugopalan, Ann. Rev. Nucl. Part. Sci. 60 (2010), 463.

3) I. Balitsky, Nucl. Phys. B 463 (1996), 99.

Y. V. Kovchegov, Phys. Rev. D 60 (1999), 034008 Phys. Rev. D 61 (2000), 074018.

4) I. Balitsky, Phys. Rev. D 75 (2007), 014001. J. L. Albacete and Y. V. Kovchegov, Phys. Rev. D 75 (2007), 125021.

5) W. Li [CMS Collaboration], J. of Phys. G38 (2011), 124027, arXiv:1107.2452

6) A. Dumitru, K. Dusling, F. Gelis, J. Jalilian-Marian, T. Lappi and R. Venugopalan, Phys. Lett. B 697 (2011), 21.

A. Kovner and M. Lublinsky, Phys. Rev. D 83 (2011), 034017, and arXiv:1109.0347

7) J. L. Albacete, N. Armesto, J. G. Milhano, P. Quiroga-Arias and C. A. Salgado, Eur. Phys. J. C71 (2011), 1705

8) J. L. Albacete and A. Dumitru, arXiv:1011.5161 [hep-ph]. J. L. Albacete, A. Dumitru and Y. Nara, J. Phys. Conf. Ser. 316 (2011), 012011, arXiv:1106.0978

9) H. Fujii, K. Itakura, Y. Kitadono and Y. Nara, J. of Phys. G38 (2011), 124125

10) A. Dumitru, H. Fujii and Y. Nara, work in progress

11) V. Khachatryan et al. [CMS Collaboration], Phys. Rev. Lett. 105 (2010), 022002.

12 ) D. de Florian, R. Sassot and M. Stratmann, Phys. Rev. D 75 (2007), 114010, Phys. Rev. D 76 (2007), 074033.

13) L. D. McLerran and R. Venugopalan, Phys. Rev. D 49 (1994), 2233, Phys. Rev. D 49 (1994), 3352.

Y. V. Kovchegov, Phys. Rev. D 54 (1996), 5463.

14) D. Boer et al., arXiv:1108.1713

15) J. L. Albacete and C. Marquet, Phys. Lett. B 687 (2010), 174.

J. Jalilian-Marian and A. H. Rezaeian, arXiv:1110.2810.

G. G. Barnafoldi, J. Barrette, M. Gyulassy, P. Levai and V. Topor Pop, arXiv:1111.3646.

16) A. Dumitru, J. Jalilian-Marian and E. Petreska, Phys. Rev. D 84 (2011), 014018.

17) C. Ciofi degli Atti, B. Z. Kopeliovich, C. B. Mezzetti, I. K. Potashnikova and I. Schmidt, Phys. Rev. C 84 (2011), 025205. 
18) M. Deak, F. Hautmann, H. Jung and K. Kutak, arXiv:1012.6037.

19) A. Mocsy and P. Sorensen, arXiv:1008.3381.

P. Sorensen, B. Bolliet, A. Mocsy, Y. Pandit and N. Pruthi, Phys. Lett. B 705 (2011), 71.

20) R. S. Bhalerao, M. Luzum and J. -Y. Ollitrault, Phys. Rev. C 84 (2011), 054901.

21) Y. Nara, arXiv:1110.2847

22) P. Tribedy and R. Venugopalan, Nucl. Phys. A 850 (2011), 136 [Erratum-ibid. A 859 (2011), 185]

23) F. Gelis, T. Lappi and L. McLerran, Nucl. Phys. A 828 (2009), 149.

24) C. Flensburg, arXiv:1108.4862

25) E. Avsar, Y. Hatta, C. Flensburg, J. Y. Ollitrault and T. Ueda, J. of Phys. G38 (2011), 124053

26) H.-J. Drescher and Y. Nara, Phys. Rev. C 75 (2007), 034905, Phys. Rev. C 76 (2007), 041903.

27) A. Bilandzic [ALICE Collaboration], AIP Conf. Proc. 1343 (2011), 465. 